\title{
La construction identitaire de l'élève par le questionnement et la discussion à visée philosophique
}

\section{Michel Tozzi}

\section{(2) OpenEdition \\ Journals \\ Édition électronique \\ URL : http://journals.openedition.org/trema/2519 \\ DOI : $10.4000 /$ trema.2519 \\ ISSN : 2107-0997 \\ Éditeur \\ Faculté d'Éducation de l'université de Montpellier}

Édition imprimée

Date de publication : 1 décembre 2010

Pagination : 8 - 22

ISSN : 1167-315X

\section{Référence électronique}

Michel Tozzi, «La construction identitaire de l'élève par le questionnement et la discussion à visée philosophique », Tréma [En ligne], 33 - 34 | 2010, mis en ligne le 01 décembre 2012, consulté le 19 avril 2019. URL : http://journals.openedition.org/trema/2519; DOI : 10.4000/trema.2519

Ce document a été généré automatiquement le 19 avril 2019

Trema 


\title{
La construction identitaire de l'élève par le questionnement et la discussion à visée philosophique
}

\author{
Michel Tozzi
}

Pour aborder cette question, qui pour nous fait référence à la pratique innovante de la Discussion à Visée Philosophique (DVP) à l'école, nous clarifierons dans un premier temps la notion d'identité (notre approche sera surtout philosophique, et non psychologique ou sociologique), puis celle de construction identitaire. Dans un second temps nous montrerons comment la pratique de la DVP en classe favorise cette construction.

\section{Identité et construction identitaire}

\section{1. Approche philosophique de l'identité}

2 La question de l'identité personnelle est

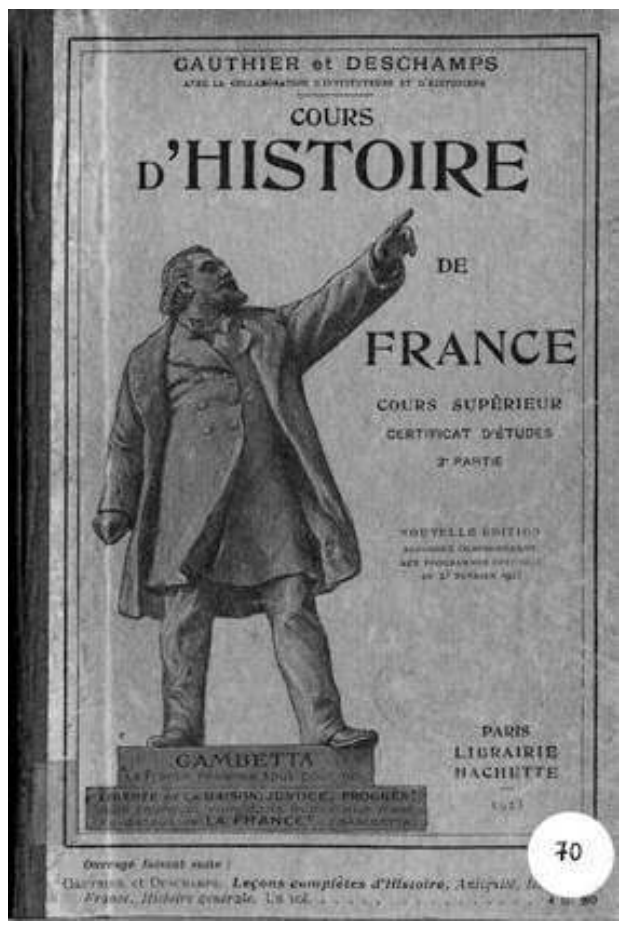
relativement récente dans l'histoire occidentale de la philosophie. Pour Aristote, ce qui caractérise un homme (et non une femme ou un esclave), c'est le fait d'appartenir à la polis, à la cité, d'être un citoyen. Dans le christianisme, la notion de personne, reprise du théâtre grec (le héros qui émerge et se distingue du chœur antique) est théologiquement refinalisée : nous sommes frères parce que fils de Dieu, et notre identité s'origine dans cette filiation divine. 
Descartes innove en fondant la subjectivité moderne: il définit l'identité de l'homme comme substance pensante : le dépassement du doute méthodique et radical de tout par la découverte qu'en doutant, il pense, l'assure du même mouvement de son existence et de son essence : il est (d'abord et surtout) une pensée, à ce moment sans corps (ce qui fonde le dualisme), mais aussi sans autrui (position solipsiste) : il fait ainsi l'économie de l'altérité dans l'identité. Y compris en soi-même, puisque la conscience cartésienne se pose dans une immédiateté massivement existentielle et ontologique, et en toute transparence par rapport à elle-même.

Hume reste au contraire dubitatif : si c'est la comparaison de mes impressions qui me permet de (me) connaître, celles-ci n'étant que diversité ne plaident guère pour un moi unifié et stable: l'unité postulée semble alors relever plutôt de la croyance (ou de l'illusion ?), que d'une certitude. Nietzsche poussera jusqu'au bout le soupçon d'un cogito brisé : le moi est une illusion grammaticale; le langage qui me fait dire je, fondamentalement trompeur, ne fait que dissimuler la poussée d'un instinct de vivre.

C'est avec Hegel que l'altérité s'invite dans l'identité. Toute conscience de soi suppose un dédoublement pour se re-connaître comme conscience. La conscience est conscience de l'autre en soi, en même temps que conscience par et pour autrui, en même temps que conscience d'autrui. Et c'est réciproque pour chacun. Qu'est-ce en effet qu'une conscience qui ne serait pas reconnue ? L'identité de chacun ne s'affirme que dans sa reconnaissance. Toute subjectivité est en ce sens une intersubjectivité.

Que ce soit dans la psychologie génétique d'un Wallon ou d'un Freud, l'identité d'un sujet s'enracine dans une origine/fondement qui le tisse d'altérité, notamment par la symbolique du langage, qui me nomme avant même que je ne sois né... Freud délogera la superbe du cogito en déplaçant le centre de gravité de notre identité profonde : de notre conscience à notre inconscient; ça parle où je croyais penser... L'identité du sujet s'opacifie, travaillée par les pulsions nouées dans un roman familial.

Qu'est-ce donc qu'une identité personnelle, celle d'un individu ou d'une personne, par opposition à celle d'un groupe?

L'identité s'éprouve, par une forme de preuve existentielle : l'expérience interne d'exister comme moi (que l'on pourra critiquer, avec ceux qui soupçonnent la valeur de l'expérience, comme illusion perceptive, ou simple croyance non fondée).

On peut la définir :

- synchroniquement (dans le présent), comme la conscience d'être soi (un individu humain, une personne, un soi qui est moi, et dis « je »), de former une unité ;

- diachroniquement (dans la durée), comme la conviction de rester soi, de garder cette unité dans la continuité (et malgré les changements).

L'identité est en ce sens mêmeté : rester le même moi quand on change, croire en un noyau qui perdure en dépit de modifications dues au temps qui passe. Ricoeur parle ici, en référence à l'un des sens latins de même, d'identité - idem. C'est une identité qui se caractérise par la stabilité dans le temps, sa robustesse. Elle peut ainsi nous définir : c'est le cas par exemple juridico - politiquement de notre état civil, qui marque notre appartenance et permet à cause de cette permanence une reconnaissance, une identification (l'identité, c'est ce qui permet d'identifier, et d'être identifié). C'est le cas aussi de notre caractère, qui nous définit psychologiquement par sa relative constance, et permet aux autres de prévoir approximativement ou assez précisément nos réactions, ce qui permet d'organiser nos interactions avec autrui.

Pour penser cette permanence du sujet, les philosophes ont utilisé des concepts. Celui de 
forme: on peut changer toutes les planches du bateau de Thésée, dit Aristote, sa "matière ", il n'en garde pas moins sa "forme " de bateau. Ou le concept de substance (Descartes) : sorte de substrat qui supporte ontologiquement le sujet dans son être, audelà de son apparaître changeant. Ou le concept plus moderne de structure, forme stable d'organisation d'éléments divers en un tout dont les parties sont en relation les unes avec les autres. On voit ici que l'identité - idem, en réduisant la temporalité à une reproduction du même en boucle, s'oppose à l'historicité, au caractère surgissant et inédit de l'événement, à l'invention temporelle du présent : elle est pour ainsi dire anhistorique...

L'identité ne se réduit pas pourtant à ce sentiment présent et durable d'être une unité qui, sauf pathologie (ex : dissociation ou dissolution de la personnalité), tient et se tient, "persévère dans son être » (Spinoza) : c'est aussi un sentiment d'unicité, d'être un « moi unique » (Stirner), original, « quelqu'un qui n'est pas quiconque» (Lacan). Mon identité peut certes se décliner par ce que j'ai de commun avec moi dans le temps qui passe, ou de commun avec d'autres (je suis, comme bien d'autres, un homme, senior, français etc.), bref par de la mêmeté partagée avec moi et autrui. Mais elle se caractérise aussi par ma singularité : ce en quoi je suis différent, différent de moi dans le temps, parce que je change physiquement et mentalement, et radicalement hétérogène et irréductible à tout autre. Cet autre aspect de l'identité, Ricoeur la nomme identité - ipse.

Dans l'identité - idem, le je est prédicable, c'est un sujet logique; on peut lui attribuer tout ce qu'il a de commun avec d'autres : Aristote est philosophe et grec, comme avant lui Platon, dont il est le disciple. Mais que peut-on dire de Socrate en tant que Socrate, puisqu'il n'a rien de commun avec quiconque en tant qu'individu singulier ? Il est un ensemble à un seul élément, indéfinissable, non prédicable, mystérieux dans son unicité...Il échappe au langage et à la logique.

Cette identité - ipse n'est pas non plus un sujet grammatical, puisque quiconque, comme moi, peut dire je. Le « je » grammatical n'est qu'un je d'emprunt au langage, une façon pour moi d'accaparer le pronom personnel de la première personne en grammaire utilisable par quiconque est quelqu'un, mais qui fait de quelqu'un un quelconque, puisqu'il est commun à tous, et non singulier pour moi. C'est encore de l'identité - idem... Si je suis singulier, à nul autre pareil, insubstituable, je suis autre que l'autre, l'autre de l'autre, et vice versa. Mon identité est personnelle et distinctive. Je ne peux donc penser mon identité sans l'altérité. Et comme on me reconnaissait en ce que j'étais le même que l'autre (européen, humain), on me reconnaît aussi et peut-être surtout parce que je suis différent.

Je suis même autre que moi, parce qu'il y a de l'autre en moi : Soi-même comme un autre dit Ricoeur. L'autre que j'ai été mais ne suis plus, que je serai peut-être mais pas encore, un inconscient en deçà de ma conscience, une surprise potentielle pour moi-même, un héros ou un salaud selon les circonstances...

\section{2. Approche philosophique de la construction identitaire}

On peut soutenir que l'identité d'un individu est essentiellement reçue, héritée, transmise. Biologiquement, on provient de vies qui nous précèdent et nous transmettent leurs gènes, et on connaît de mieux en mieux l'influence des gènes sur notre avenir physique, et en partie mental. Juridiquement nous héritons d'une identité nationale par la loi du sol ou $\mathrm{du}$ sang. Psychologiquement, nous sommes façonnés par nos relations parentales premières, qui marquent et structurent notre inconscient. Sociologiquement, nous sommes imbibés par notre milieu géographique, social, culturel, idéologique, par 
l'éducation, la langue d'origine, les médias, tout notre environnement, qui génèrent à notre insu des habitus («Dis-moi la catégorie socioprofessionnelle de ton père, dit le Bourdieu des années 1970, je te dirai avec une forte probabilité ta place future sur le marché du travail »).

Dans une perspective matérialiste déterministe, je suis produit plus que processus, agent plus qu'acteur. Et dans une perspective religieuse fataliste, tout est déjà écrit de ma vie ; par exemple la grâce de Dieu ne dépend pas de mes œuvres. Dans ces scénarii, le cosmos par le jeu de l'enchevêtrement de ses causes, ou Dieu, aux desseins souvent impénétrables, sont à l'origine du roman de ma vie, dont je ne suis à mon insu que le personnage imposé.

On a là une identité héritée et prédictive, de l'ordre de la trajectoire, au sens balistique : une énergie est donnée au départ, vers un but prédéterminé. Simplement, j'ignore ces déterminations, dans l'illusion d'être libre (comme la pierre qui dévale une pente, si elle avait une conscience, pourrait se croire libre de se mouvoir, dit Spinoza). Si l'on veut encore parler de "construction", c'est au sens où va se dérouler dans le temps une histoire, mon histoire, mais elle est déjà programmée: elle n'existe au départ que virtuellement, mais va actualiser un potentiel balisé et circonscrit. Les jeux du je sont faits. Il y a là quelque chose de l'ordre de la production, de la reproduction, de la répétition, du même... Je ne puis être que ce que je pouvais être, et ne peut excéder mes conditions de possibilité.

Mais peut-on parler de construction, quand le chemin emprunté est déjà prévu ou prévisible? La notion, inspirée du bâtiment, suppose certes un plan qui l'antécède, mais toute la question est de savoir qui fait ce plan (le hasard, la causalité, Dieu...), et s'il peut ou non changer, plus crucialement, si je peux changer de plan de vie en cours de route...

Il y a des philosophes comme Sartre pour soutenir en contrepoint que notre liberté est absolue, inconditionnée, «que nous n'avons jamais été aussi libres que sous l'occupation allemande ", que notre existence excède toute essence qui réifierait le jaillissement de notre liberté. Il nous rappelle qu'une contrainte n'est peut-être jamais absolue, et qu'une détermination n'est peut-être jamais un déterminisme strict. On sait par exemple qu'un gène n'influence qu'en interaction avec un environnement, ce qui relativise ses effets par cette contextualisation; qu'un résilient dément les prédictions de son échec; que si le sexe a une composante biologique essentielle, il est aussi la construction sociale d'un "genre »; que la thérapie permet de revenir sur un passé trop déterminant etc. Toute identité humaine a une histoire, qui porte sa part de contingence au-delà du probable et du nécessaire, à cause du surgissement d'événements qui dérangent les voies les plus tracées...

Toute subjectivité est en effet inscrite dans une temporalité, qui en fait une étoffe en train de se tisser. Cette temporalité prend l'allure d'un récit où je suis un personnage en intrigue avec tout ce qui m'arrive dans la vie. Mon identité personnelle est donc, comme disent P. Ricoeur ou J. Bruner, narrative.

- La mémoire est une façon de se raconter son passé, de dénouer et renouer les fils. Elle n'est pas simple enregistreur impartial de faits objectifs, mais sélection affective impliquant oubli, refoulement et souvenirs vivaces. Elle est en partie fictionnelle, elle construit et reconstruit a posteriori en permanence du sens, qui change avec le temps, réévalue les événements avec le recul.

- Quand au projet, il est une façon de se raconter ce que l'on veut dans l'avenir, un récit anticipé, qui va du rêve irréaliste à la planification raisonnée.

On a vu que certains pensent que ce récit est déjà programmé. 
On peut aussi penser, sans minimiser toutes les contraintes de nos déterminations diverses, que nous pouvons être acteur d'une pièce que nous n'avons pas choisie, avec l'improvisation du jeu que donne l'interprétation d'un rôle: c'est le point de vue des stoïciens. Nous n'avons pas choisi d'être né, mais nous pouvons trouver que c'est une bonne (la joie d'exister de Spinoza), ou une mauvaise chose ("l'inconvénient d'être né » selon Cioran).

Peut-être même que nous pouvons plus, en dépit de nos déterminations : devenir acteur de notre vie, par la réflexion, la délibération et le choix, l'initiative, l'action dont nous nous sentons responsable et comptable devant autrui et nous-même. C'est l'option de Ricoeur. L'homme est certes pris dans le réseau intriqué des causes efficientes, où toute action a une ou plusieurs causes dont elle est l'effet. Mais il possède en propre la capacité d'une causalité téléologique, où le but poursuivi précède le moyen qui permettra de l'atteindre, où la fin existe avant la cause qui la rendra possible, ce qui permet à l'homme de prendre l'initiative de " commencer dans le monde ». Cette liberté humaine s'éprouve dans le sentiment, certitude fragile mais au-delà de tout soupçon, d'attestation de son existence, et d'assomption de ses actes. Parce que nous écrivons notre vie comme un récit, parce que notre identité est narrative, elle implique une dimension éthique, qui est le cour de l'identité proprement humaine.

La construction identitaire personnelle nous semble donc un processus historique au croisement de déterminations diverses réelles, qu'il serait vain de nier, et d'une liberté de penser et d'agir en homme responsable.

Résumons-nous : l'identité personnelle est celle d'une subjectivité. Cette subjectivité est une intersubjectivité, car pétrie d'altérité. Elle se construit dans une temporalité. On ne doit pas seulement penser l'histoire de cette construction, notamment chez l'enfant et l'adolescent, en terme psychologique de développement, mais dans une problématique philosophique: dialectique entre le même et l'autre, la continuité et le changement, le déterminisme et la liberté.

Insistons sur deux éléments essentiels de cette construction :

- L'altérité logée au cœur de ma subjectivité vient à moi et me constitue tout particulièrement dans et par le langage, qui m'inscrit dans le symbolique, espace intersubjectif de l'intercompréhension.

- Ce qui permet la construction identitaire de l'élève ou de l'enfant en tant que sujet pensant, c'est la capacité de réflexivité dont le langage humain est porteur. Il n'y a en effet pas de pensée construite sans langage.

La DVP, comme dispositif scolaire et dans la cité (café philo, etc.) réalise, dans la perspective de cette construction identitaire, un heureux nouage entre confrontation $\grave{a}$ l'altérité plurielle incarnée (par la discussion), échanges langagiers (par la parole), et communauté réflexive (par la pensée)...

\section{La construction identitaire par et dans une Discussion à Visée Philosophique (DVP)}

6 Nous allons ici nous restreindre au cadre scolaire, en particulier de l'école primaire. En quoi la pratique de la Discussion à Visée Philosophique favorise-t-elle la construction identitaire de l'élève et de l'enfant comme sujets parlants - pensants ? Nous répondons : par une praxis langagière, par une praxis réflexive, et par un double processus de socialisation, à la fois démocratique et socio - cognitif, ces processus étant étroitement 
interdépendants. On trouvera ci-dessous un schéma formalisant cet ensemble, que je me bornerai à commenter.

\section{1. Langage et pensée dans la discussion}

7 La DVP est la rencontre entre la parole orale de sujets et leur pensée réflexive. C'est cette rencontre qui favorise la construction de leur identité langagière (leur "parlêtre" comme dit Lacan), et de leur identité réflexive (leur "pensêtre », leur identité d'être pensant). Dans le processus psychogénétique général de co-développement du langage et de la pensée, la discussion entre pairs réglée par le maître utilise ici le code oral du langage dans sa forme d'une langue naturelle (le français), comme mode d'élaboration et d'expression d'une pensée par et dans le langage. L'objet de travail proposé au groupe dans une DVP étant le plus souvent une question prenant sens dans le vécu des enfants mais assez abstraite, par sa généralité (voire l'universalité) qui concerne la condition humaine, l'échange est de nature réflexive: c'est une question qui fait problème aux hommes. D'où l'intérêt de «s'y coller» ensemble. Il y a devant une question problématique une nécessité de réfléchir face à la difficulté ou la complexité de la question avant de répondre, ou de débattre à cause des différents points de vue qui vont s'exprimer.

L'usage de la langue dans ce type d'échange est donc réflexif, et non fonctionnel, ce qui introduit les interlocuteurs dans un autre rapport à la langue que pragmatique (ne plus seulement parler pour agir au quotidien sur le monde). On constate de ce fait un registre de langue plus soutenu, car on cherche le mot ou l'expression au plus près d'une pensée qui se cherche elle-même par et dans le langage. Parler pour penser, pas seulement pour dire, et penser en parlant, car il n'y a guère de pensée (en tout cas de pensée réflexive) sans langage. La discussion apprend du même mouvement à parler et à penser. Le langage n'est pas un préalable à la pensée (même s'il en est une condition, et si on peut penser plus précisément avec un langage plus développé), car ils se co-développent : travailler sa pensée c'est en effet du même coup travailler la langue, et travailler la langue, c'est se donner le moyen de mieux penser. Car la pensée, c'est la mise au langage d'une expérience, et tout jeune, l'enfant a déjà une appréhension du monde...

La DVP peut donc commencer très tôt, dès que l'enfant apprend à parler, parce que parler c'est entrer en interaction avec autrui ( $F$. François parle même de la nature argumentative du langage); parler, c'est déjà penser avec les mots des autres : toute langue dans sa dimension culturelle est porteuse d'une certaine vision du monde. Mais parce qu'aussi toute parole d'un individu traduit une expérience du monde distinctive, singulière, qu'elle porte au langage dans son altérité.

Il peut y avoir discussion dès que l'enfant commence à s'exprimer, à exprimer une idée, à écouter et comprendre la réaction d'un autre à sa propre parole, à répondre à ce qu'il a compris de la parole des autres pour réagir, adhérer, objecter, interroger... Nous y travaillons pour notre part dès la grande section de maternelle.

\section{2. Construction identitaire et pensée réflexive}

8 Qu'est-ce qu'une pensée ? Se demande Descartes dans son enquête philosophique sur luimême. Et de décliner une substance qui «sent, imagine, conçoit». Et une pensée réflexive? Celle qui réfléchit, c'est-à-dire use de son entendement. C'est une démarche rationnelle, qui peut être aussi bien d'ordre scientifique que philosophique. Ce dernier 
type utilise seulement pour se déployer la langue naturelle, et non des procédures formalisées (comme en mathématique par exemple). L'administration de la preuve se fait sous forme notamment d'argumentation, et non de processus de validation faisant appel à la démonstration mathématique ou à l'expérimentation physique ou biologique.

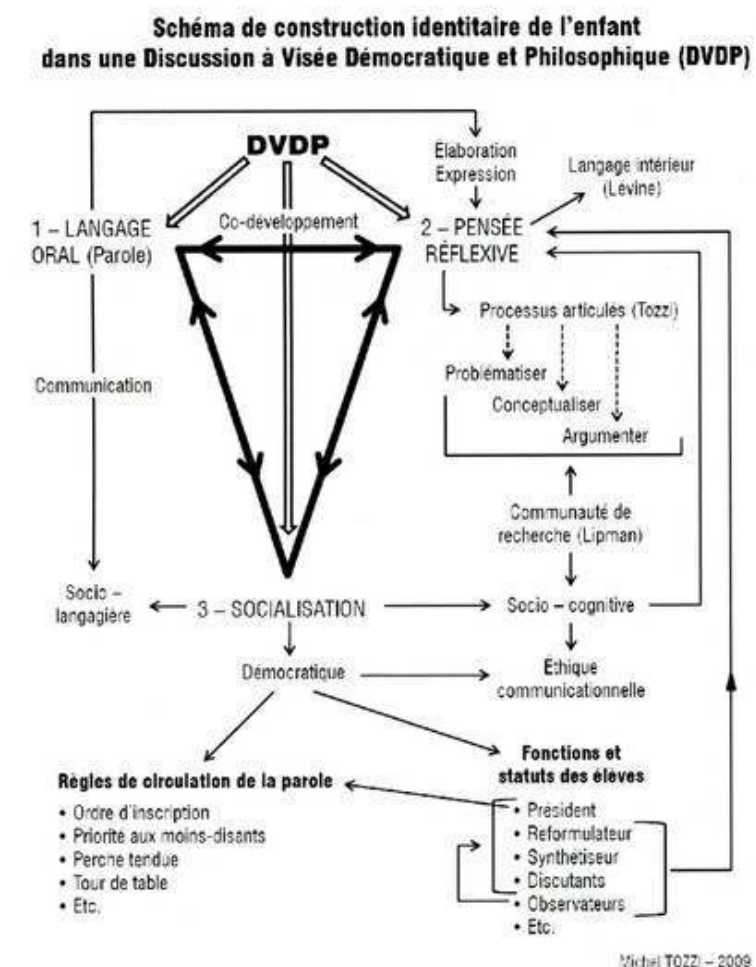

La DVP contribue à la construction identitaire du sujet comme être pensant par l'apprentissage du philosopher, c'est-à-dire de processus interdépendants de problématisation de questions concernant le sens de la condition humaine, de conceptualisation de notions abstraites permettant de les poser et les résoudre, d'argumenter ou de critiquer rationnellement leur réponse, dans une visée de vérité, ou de recherche d'une « vie bonne dans des institutions justes » (Ricoeur).

Cette construction passe chez les enfants, avec ou sans support au départ (littérature de jeunesse, mythes ...) : par l'expression de questions qui sont les leurs («Une amitié, ça dure toujours?»); par la mise en relation de ces questions avec leur propre expérience (ici de l'amitié), en s'appuyant sur des exemples et des contre-exemples; par la recherche de précision et de définitions des mots employés («Un ami, c'est quelqu'un à qui on confie ses secrets »); par l'élaboration de distinctions conceptuelles (ami, copain, amoureux, ça se ressemble mais ce n'est pas pareil); par l'affirmation d'accords ou de désaccords dont ils doivent donner les « bonnes raisons » etc. On devine le rôle essentiel de l'enseignant dans l'animation d'une DVP. C'est par ses exigences intellectuelles pour que de tels processus soient mis en oeuvre dans l'échange que les enfants développent des compétences à problématiser, conceptualiser et argumenter, qui vont le doter d'outils intellectuels pour « penser par lui-même ».

\section{3. Une activité socialisatrice}

10 La discussion est une activité langagière de communication entre humains. Il s'agit d'entrer en interlocution, de comprendre et se faire comprendre, de créer une intersubjectivité à 
base d'intercompréhension (Habermas). Elle confronte à l'altérité plurielle: on apprend à vivre ensemble en discutant. Elle est en ce sens socialisatrice, productrice d'identité collective et individuelle par l'échange.

La socialisation a été pensée par les psychologues et les sociologues soit comme une intériorisation par l'individu des normes et comportements collectifs (l'individu étant alors premier: ex Durkheim), soit par une singularisation individuelle à partir d'une indifférenciation première, comme un processus d'individualisation individuel et social (Freud, Wallon). C'est l'apprentissage par des humains du vivre ensemble, dans lequel l'éducation va jouer un rôle déterminant. Et l'on sait à quel point ce processus est devenu problématique dans la société moderne individualiste, où l'individu revendiquant fortement d'être reconnu dans sa singularité, peine à partager des valeurs communes. D'où la préoccupation scolaire de la « citoyenneté » (il faudrait plus exactement parler de civilité).

\section{3. 1. La DVDP comme socialisation démocratique}

11 Certaines conditions sont nécessaires à cet apprentissage si l'on veut l'ordonner à une finalité civilisatrice. Par exemple si et quand on veut que la discussion soit démocratique. Il $\mathrm{y}$ faut un espace circulaire : on discute face à face, comme les guerriers dans l'Iliade qui se mettent en cercle, et déposent leurs armes («rompre les lances»), en avançant pour parler chacun à son tour puis en reprenant leur place sur le périmètre commun ; des règles de tours de paroles, pour réguler le toujours possible rapport de force: ordre d'inscription, priorité à ceux qui ne se sont pas encore exprimés ou parlent peu, tours de table; et des attitudes favorables à un véritable échange : discuter, c'est surseoir à la violence physique (la parole est toujours à distance du contact), mais aussi verbale (dans l'injure, on se dispute, on ne discute pas ; dans la moquerie, on disqualifie l'autre comme interlocuteur non valable); c'est écouter (position d'accueil) pour comprendre (effort pour pénétrer dans la vision de l'autre), répondre après que l'autre ait fini d'intervenir (patience de l'écoute et maîtrise des affects), car le droit de parler de chacun suppose pour être exercé le silence de tous quand un seul parle etc.

La DVP est ainsi pour nous une DVDP (Discussion à Visée Démocratique et Philosophique), qui contribue à l'éducation civile et civique des élèves, dans un « espace scolaire public de discussion", qui développe dans ce lieu protégé par des règles une "laïcité de confrontation » (Ricoeur), et non d'indifférence. Le caractère démocratique des règles et des fonctions déléguées par le maître (un élève président de séance est le garant de l'application des règles) permet l'expression de chacun (il n'y a pas d'élèves plus " égaux " que d'autres par rapport au droit d'expression, même si tous ne s'expriment pas de fait), et du minoritaire (ce qui assure une pluralité d'opinions, condition pour qu'il y ait débat, qu'arrête le consensus). Il y a aussi le souci du muet ou du petit parleur par la perche tendue par le président, car on a idéalement besoin de la parole de tous pour enrichir l'échange (on peut aussi lire ce qu'on a écrit quand on est sollicité). Mais il y a un droit de se taire, pour ne pas tomber dans le genre scolaire de «l'interrogatoire » magistral à la réponse contrainte de l'élève. L'intérêt d'observateurs est ici de se préparer pour la fonction de président, de mesurer la complexité et la difficulté de mettre en oeuvre son cahier des charges, de proposer aussi des améliorations de fonctionnement dans un esprit d'entraide, car l'esprit de la discussion est coopératif. La délégation de l'autorité à des élèves est responsabilisante pour ceux-ci, qui peuvent étonner par leur sérieux à exercer une fonction nécessaire au fonctionnement de l'activité qu'on leur donne avec confiance. 
Tout cela contribue à la construction d'une identité citoyenne, par la prise de parole réglée pour exprimer publiquement des opinions, et un rapport coopératif à la loi.

\section{3. 2. La DVP comme socialisation réflexive}

Mais la spécificité de la DVP est de favoriser un certain type de socialisation: une socialisation réflexive. L'intérêt d'une socialisation réflexive dans un contexte individualiste est de contribuer à atténuer l'effet d'affaiblissement du lien social (ce qui fait tenir ensemble une société, et ici son école).

Mais qu'entendons-nous par socialisation « réflexive » dans la DVP ? Une façon de « vivre ensemble en discutant philosophiquement ». Epicure appelait cela "l'amitié philosophique ", forme d'excellence de la philia. Pourquoi y a-t-il socialisation? Parce qu'on apprend à discuter ensemble sans se disputer. A être et souvent demeurer en désaccord mais dans la paix civile, et non la guerre. Faire l'expérience du désaccord dans la paix est éminemment civilisateur. C'est accepter et même vouloir la différence, car elle enrichit au lieu de diviser.

J. Lévine parlait, dans cet apprentissage de la pensée possible dès l'école maternelle, de "langage intérieur", activité par laquelle l'enfant dit et se dit dans l'atelier philo son rapport à autrui et au monde. Cette activité socio - langagière produit de la socialité, de la sociabilité, parce que l'enfant est institutionnalisé comme « interlocuteur valable » dans la DVP, écouté par un maître qui s'intéresse à ce qu'il dit, tout dans l'écoute puisqu'il n'intervient pas lui-même, et dégage volontairement, par son silence, un lieu et moment d'expression individuelle de chacun. L'enfant s'autorise alors, parce qu'il y est librement autorisé, à entrer dans l'humanitude des êtres parlants - pensants. Cet effet de « reconnaissance » (au sens de A. Honneth ou de P. Ricoeur) instaure le " petit » dans une dignité d'homme, dont on observe les effets (re)narcissisants avec des enfants ou adolescents en difficulté scolaire et personnelle (école primaire en zone sensible ou Segpa en collège par exemple). Chacun reprenant consistance de sujet valable (qui a une valeur humaine) dans un dispositif « contenant ", voyant ce statut de sujet confirmé par et dans une parole qui ose penser et se sent entendue, un apaisement s'instaure dans le " groupe cogitans ». Le langage, d'invective spontanée en réaction à un affect blessé (l'injure), médiation intérieure entre le trouble ressenti et le coup prêt à partir, devient à nouveau communication humaine, parce qu'il élabore et exprime une pensée, au lieu d'une agression (celle de l'agressé agresseur).

L'exercice de la réflexivité s'exerce aussi dans un moment métacognitif après chaque discussion, où les élèves (en tant que "praticiens réflexifs »), analysent comment ils ont exercé le cahier des charges de leur fonction, les difficultés auxquelles ils ont été confrontés, les moyens qu'ils ont pris ou non pour les surmonter. Les observateurs s'entraînent explicitement à cette analyse, qu'ils renvoient dans un second temps au groupe.

Dans la DVP, l'horizon d'une vérité partageable parce que rationnelle engage dans la discussion, parce qu'on en escompte un consensus. On rencontre alors le désaccord, qui reste un scandale intellectuel pour qui croit détenir la vérité. Il faut donc qu'on s'en explique, dès que l'on croit l'autre de bonne foi. Mais pas pour être le plus fort. Supposons un instant que l'autre ait raison : un peu, beaucoup... Que penser alors?

L'enjeu c'est la question, son élaboration et sa réponse, et non d'avoir raison de l'autre. Car il s'y joue du sens, et non plus des rapports de force. Nous y sommes tous impliqués, car les questions philosophiques sont celles de tous, donc de chacun. Personne n'est en 
droit hors jeu. Tout le monde a intérêt dans la DVP au jeu de la question, « pour de vrai et de bon ", car il y va des raisons de vivre et de mourir : un jeu très sérieux, qui ne va pas sans règles.

Considérer que ma réponse est une croyance ferme, mais peut-être pas une vérité absolue et définitive. Les questions philosophiques sont urgentes, mais difficiles et complexes. Déjà pour bien les poser et les comprendre. A fortiori pour y répondre. Cela ouvre un monde des réponses possibles, largement labouré par l'histoire de la philosophie... Être donc capable de remettre en question ce que je pense, et apprendre à être interpellé par la différence, la divergence, l'objection. Prendre l'autre au sérieux: partenaire, non adversaire. Interlocuteur véritable, qui peut m'apporter en me déstabilisant, me déplaçant, en ami exigeant. Quoi qu'il en coûte de mon orgueil, de la « préservation de ma face " (Goffman). Donner à ma thèse le statut d'hypothèse à faire (in)valider par le groupe.

Il y a là à la fois une morale de la pensée (se donner les conditions de penser bien et mieux) et une éthique communicationelle (Habermas) : le groupe est un « intellectuel collectif » qui constitue une "communauté de recherche" (Lipman), où chacun est une personneressource intellectuelle pour tous les autres. L'éthique communicationnelle, c'est beaucoup plus que de la "civilité », où l'on ne doit pas couper quelqu'un qui parle, se moquer, etc.

Exemplifions : un président démocratique ne privilégie dans les tours de parole ni ceux qui sont en face par rapport à ceux qui sont sur les côtés (il apprend à regarder le groupe) ; ni ses amis, ou ceux dont il a peur (la règle est anonyme, et il n'y en a pas de plus "égaux» que d'autres). De même, un élève qui reformule un camarade (le « reformulateur »), c'est quelqu'un qui montre qu'il prend en compte la pensée de celui-ci par une écoute intellectuellement exigeante pour comprendre et éthiquement fidèle pour ne pas trahir une parole dite : il respecte à la fois la personne et sa pensée, la personne à travers sa pensée. Dans un effort de décentration psychologique, il parle comme s'il était l'autre ; dans un effort éthique d'honnêteté, surtout s'il est en désaccord avec ce qui a été dit, il résiste à sa propre résistance, en parlant du lieu de son désaccord. Et c'est encore plus exigeant intellectuellement et éthiquement pour celui qui fait le compte rendu ou la synthèse de la discussion (le "synthétiseur »), parce qu'elle porte sur plusieurs interventions. C'est pourquoi le reformulateur et le synthétiseur ne doivent pas intervenir dans le débat, pour concentrer toute leur énergie sur l'écoute.

Ce qui produit une relative sérénité dans la dynamique du groupe, c'est l'enjeu de s'exprimer sur des questions choisies par les élèves eux-mêmes, importantes pour leur vie, et d'entendre différents points de vue sur les problèmes qu'elles soulèvent. La dynamique n'est pas seulement argumentative, mais problématisante et conceptualisante. Quand on se place devant une question, on est en situation de recherche, et pas immédiatement d'affirmation. Idem quand on cherche à définir un mot, qui renvoie à une notion. Il n'y a pas dans ces cas affrontement de positions, mais interrogation, essai, et il est surprenant par rapport à des représentations habituelles du débat (comme combat!) de voir un groupe chercher, dans un langage très modalisé par le questionnement ou la clarification des concepts, dans une attitude (pour reprendre des concepts d'Aristote), plus heuristique qu'éristique...

Quand s'esquissent des réponses à des questions posées (en philosophie on dit des thèses), la confrontation est sociocognitive, et l'animateur doit veiller à ce qu'elles ne dégénèrent pas en conflits socio - affectifs, portant sur des personnes et non des idées... La psychologie sociale de l'apprentissage (PERRET - CLERMONT, DOISE, MUGNY, CARUGATI 
etc.) a montré à quel point le conflit sociocognitif, à certaines conditions, amène l'élève à travailler critiquement ses représentations premières (les "opinions" ou préjugés en philosophie), et à progresser dans sa conception du monde. VYGOTSKI de son côté a montré comment la confrontation interindividuelle favorise le débat intra individuel. En décentrant l'enfant de son opinion par la rencontre avec l'altérité, la discussion lui apprend à penser, c'est-à-dire à se mettre du point de vue de l'autre pour penser avec luimême, avec et contre cet autre en lui : «La pensée est le dialogue de l'âme avec ellemême » disait déjà Platon. On voit ici à l'œuvre le processus de socialisation sociocognitif que met en œuvre la DVP.

C'est dans la famille, puis dans l'école que l'enfant construit son identité d'enfant et d'élève. La DVDP contribue à cette construction dans une triple dimension: de façon langagière, puisque la discussion développe des compétences de maîtrise orale de la langue dans une interaction sociale soutenue; de façon réflexive, ce qui est philosophiquement déterminant, en développant sous la conduite du maître des compétences de problématisation, de conceptualisation et d'argumentation rationnelle à visée universalisante. Ce processus de construction d'être parlant/pensant du " petit d'homme » est du même mouvement socialisateur, puisqu'on y apprend à vivre ensemble dans et par la coopération discussionnelle : démocratiquement socialisateur par le type de dispositif retenu (fonctions déléguées et règles de fonctionnement); et socialisateur par la réflexion commune engagée dans une communauté de recherche à visée philosophique... Une contribution éducative à une citoyenneté réflexive...

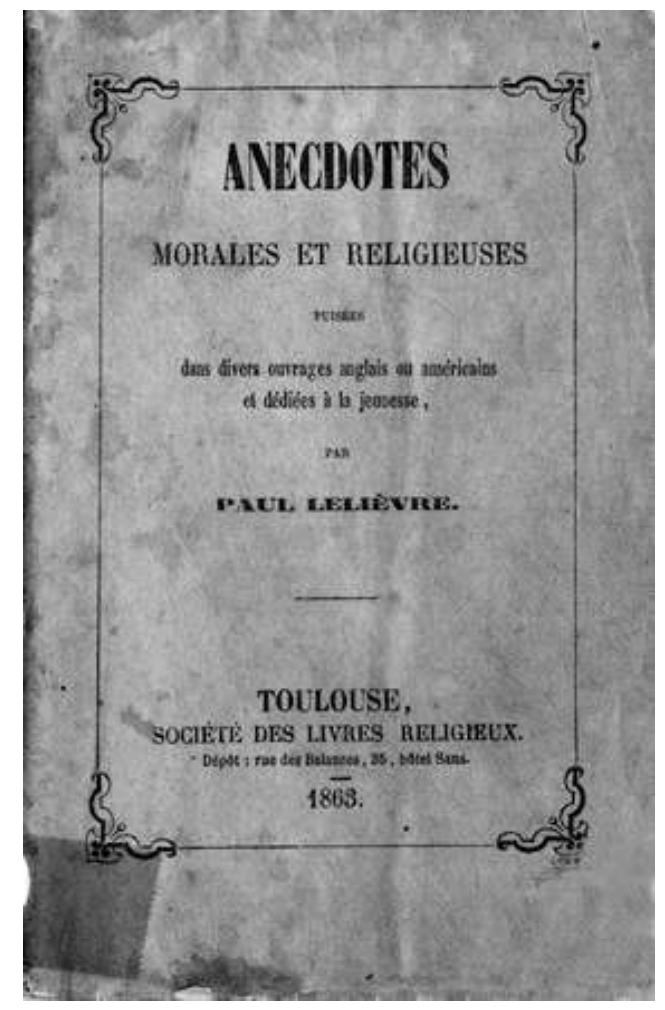

Image tirée de Anecdotes morales et religieuses / Paul Lelièvre. Toulouse : Société des livres religieux, 1863. 301 p. ; 15 cm. - Couverture. Source : CEDRHE. 


\section{BIBLIOGRAPHIE}

POUR LES ARTICLES, VOIR MON SITE : $\underline{w w w . p h i l o t o z z i . c o m}$

POUR MA REVUE EN LIGNE : Diotime, PUBLIÉE PAR LE SCEREN-CRDP DE MONTPELLIER www.crdp-montpellier.fr/ ressources/agora/

Ouvrages de l'auteur de l'article

2001 (coord.) : L'éveil de la pensée réflexive à l'école primaire, Hachette-Crdp de Montpellier

2002 (coord.) : La discussion philosophique à l'école primaire - Pratiques, formations, recherches, Crdp de Montpellier

2002 : (coord.) Nouvelles pratiques philosophiques en classe, enjeux et démarches, Crdp de Bretagne

2003 : (coord.) Les activités à philosophique en classe, l'émergence d'un genre ?, Crdp de Bretagne

2004 : La discussion en éducation et formation (coord. avec R. ETIENNE), l'Harmattan

2005 : Penser par soi-même, 6ième édit., Chronique sociale, Lyon

2006 : Débattre à partir des mythes à l'école et ailleurs, Chronique sociale, Lyon

2007 (coord.) : Apprendre à philosopher en discutant : pourquoi et comment ? De Boeck, Bruxelles, Belgique

2007 (Rédaction du Chap.1 sur l'école primaire) : La philosophie à l'école primaire dans le monde, Unesco. Téléchargeable sur : unesdoc.unesco.org/images/0015/001536/153601F.pdf

2008 : La littérature en débats : discussions à visée littéraire et philosophique à l'école primaire (avec Y. SOULE et D. BUCHETON), Sceren-Crdp de Montpellier

\section{RÉSUMÉS}

Pour aborder cette question, qui pour nous fait référence à la pratique innovante de la Discussion à Visée Philosophique (DVP) à l'école primaire, nous clarifierons dans un premier temps la notion d'identité, par une approche non psychologique ou sociologique, mais référée à des auteurs philosophiques; puis celle de construction identitaire, pensée en relation avec les notions de subjectivité, de temporalité, de langage et de liberté. Dans un second temps, nous montrerons comment la pratique de la DVP en classe favorise cette construction de l'enfant en tant que sujet pensant : en premier lieu, à travers un usage réflexif, et non fonctionnel, de la langue ; en second lieu par la mise en oeuvre d'un certain nombre de démarches de pensée à visée philosophique ; enfin par un processus démocratiquement socialisateur.

To address this issue which in our view refers to the emerging/innovating practice of dialogical philosophical discussion in elementary school, we will first clarify the notion of identity through an approach which is neither psychological nor sociological but in reference to philosophical authors; and then the notion of the construction of identity viewed in relation to the notions of subjectivity, temporality, language and liberty. Secondly, we will show how dialogic discussion 
in class fosters the child's construction as a critical thinking subject : first, through a reflective but not functional use of language; second, through the implementation of various dialogical discussions and finally through a democratic process facilitating socialization.

INDEX

Mots-clés : discussion à visée philosophique, identité, philosophie, socialisation

\section{AUTEUR}

MICHEL TOZZI

Université Montpellier III 